(2) OPEN ACCESS

\title{
Age independent survival benefit for patients with hepatocellular carcinoma (HCC) without metastases at diagnosis: a population-based study
}

\author{
Enrico N De Toni (1) , ${ }^{1}$ Anne Schlesinger-Raab, ${ }^{2}$ Martin Fuchs, ${ }^{3}$ Wolfgang Schepp, ${ }^{3}$ \\ Ursula Ehmer, ${ }^{4}$ Fabian Geisler, ${ }^{4}$ Jens Ricke, ${ }^{5}$ Philipp Paprottka, ${ }^{6}$ Helmut Friess, ${ }^{7}$ \\ Jens Werner, ${ }^{8}$ Alexander L Gerbes, ${ }^{1}$ Julia Mayerle, ${ }^{1}$ Jutta Engel ${ }^{2}$
}

- Additional material is published online only. To view please visit the journal online (http://dx.doi.org/10.1136/ gutjnl-2018-318193).

For numbered affiliations see end of article.

\section{Correspondence to}

Dr Enrico N De Toni, Department of Internal Medicine 2 University of Munich, Muenchen 80333, Germany; enrico.detoni@med.unimuenchen.de

ENDT and AS-R contributed equally.

Received 28 December 2018 Revised 12 February 2019 Accepted 21 February 2019 Published Online First 16 March 2019

\section{ABSTRACT}

Objective Hepatocellular carcinoma (HCC) is a major cause of death worldwide and its incidence is expected to increase globally. Aim of this study was to assess whether the implementation of screening policies and the improvement of treatment options translated into a real-world survival benefit in $\mathrm{HCC}$ patients.

Design 4078 patients diagnosed with HCC between 1998 and 2016 from the Munich Cancer Registry were analysed. Tumour characteristics and outcome were analysed by time period and according to age and presence of metastases at diagnosis. Overall survival (OS) was analysed using Kaplan-Meier method and relative survival (RS) was computed for cancer-specific survival. Cox proportional hazard models were conducted to control for prognostic variables.

Results While incidence of HCC remained substantially stable, tumours were diagnosed at increasingly earlier stages, although the median age at diagnosis increased. The 3 years RS in HCC improved from $19.8 \%$ in $1998-$ $2002,22.4 \%$ in $2003-2007,30.6 \%$ in 2008-2012 up to $31.0 \%$ in 2013-2016. Median OS increased from 6 months in 1998-2002 to 12 months in 2008-2016. However, analysis according to the metastatic status showed that survival improved only in patients without metastases at diagnosis whereas the prognosis of patients with metastatic disease remained unchanged. Conclusion These real-world data show that, in contrast to the current assumptions, the incidence of $\mathrm{HCC}$ did not increase in a representative German region. Earlier diagnosis, likely related to the implementation of screening programmes, translated into an increasing employment of effective therapeutic options and a clear survival benefit in patients without metastases at diagnosis, irrespective of age.

\section{INTRODUCTION}

Hepatocellular carcinoma (HCC) is the second most common cause of cancer-related death worldwide, and its incidence is expected to rise further. ${ }^{1}$ However, this trend might not reflect the reality of single states or regions, due to the different prevalence of risk factors, which account for a wide variability of incidence. ${ }^{2}$ In addition, estimations of the incidence of HCC could be affected by other factors, including the employment of different

\section{Significance of this study}

What is already known on this subject?

- Hepatocellular carcinoma (HCC) is the second most common cause of cancer-related death worldwide and its incidence is thought to rise further on a global scale.

- In recent years, several efforts have been conducted to improve HCC-mediated mortality: these include the development of direct antiviral drugs for $\mathrm{HCV}$, the implementation of secondary prevention screening programmes, the application of algorithms for therapy stratification and the improvement of local and surgical therapies.

- However, whether and to which extent these advances have translated into a clinical benefit in the general population is a matter of debate.

What are the new findings?

- We found that, in contrast to the current assumptions, the incidence of HCC over the last 18 years remained unchanged in Germany.

- Furthermore, tumours were diagnosed at a progressively earlier stage and median survival doubled between 1998 and 2016.

- This is due to earlier tumour stage at diagnosis independently of the improvement of treatment modalities.

- To our knowledge, this is the first study showing that the advances in the prevention and treatment of HCC, well documented in clinical studies, translated into a survival advantage for HCC patients in the general population.

How might it impact on clinical practice in the foreseeable future?

- Our findings confirm that early diagnosis is a major factor influencing survival of HCC patients and highlight the need for a consistent implementation of surveillance programmes to further reduce HCC-related mortality.

diagnostic criteria and biases influencing reports from tertiary care centres, likely reflecting changes in referral rates rather than in real incidence. ${ }^{3-5}$

In recent years, several efforts have been made to improve HCC-related mortality: vaccination 
against $\mathrm{HBV}$ is widespread worldwide, ${ }^{6}$ treatment of chronic HCV has considerably improved ${ }^{7}$ and surveillance programmes have been included in international guidelines since the early 2000s and have been increasingly implemented. ${ }^{89}$

Furthermore, the enactment of therapeutic algorithms and the advances in treatment options, including the definition of prognosis-based criteria for liver transplantation, ${ }^{10}$ the more recent advent of effective systemic treatment ${ }^{11}$ and the implementation of interdisciplinary decision-making processes are thought to have contributed to increasing the survival of HCC. ${ }^{12}$ However, due to the different availability and accessibility of screening programmes and therapeutic options among different countries and centres, ${ }^{13}$ it is not known whether and to which extent early diagnosis and the advances in the treatment of HCC documented by clinical studies translated into a clinical benefit for the general population. ${ }^{14} 15$

In this study, we aimed at assessing incidence and survival of HCC in a German population and the influence of the factors determining their change over the last 20 years. To this scope, data from a population-based cohort of patients diagnosed with HCC between 1998 and 2016 were analysed.

\section{METHODS}

\section{Data collection}

The Munich Cancer Registry (MCR) is a population-based clinical cancer registry in Southern Germany comprising the region of Upper Bavaria and part of Lower Bavaria. Its catchment area, which comprised 2.3 million inhabitants in 1998, was extended to include 3.7 million inhabitants in 2002 and 4.7 million in 2007. Since then, the population increased to the actual 4.8 million inhabitants without further extensions of the catchment area. Pathology reports of solid tumours from all pathology laboratories in the catchment area are systematically sent to the MCR. Additional data are provided by clinicians who fill up online standardised forms comprising patients' residence and age, along with other primary disease characteristics such as tumour, node, metastases (TNM) stage, histology, grade as well as therapies received. The life status of these patients is systematically maintained on the basis of death certificates. The proportion of patients recorded only owing to the availability of a death certificate (death certificate only [DCO] cases) is usually high in HCC and averages at 28.3\% between 1998 and 2015 with a tendency to an improving development. ${ }^{16}$ Data on the age distribution of the German population in the last 20 years were obtained from the publically available database of the Federal Statistical Office (Destatis) and from the Bavarian State Office for Statistics. ${ }^{17} 18$

\section{Patients}

Seven thousand three hundred and sixteen patients with residence in the catchment area were diagnosed between 1998 and 2016 with liver cancer (figure 1). Patients with intrahepatic biliary tumours, sarcoma, lymphoma and fibrolamellar HCC were excluded from analysis. After exclusion of 2061 patients with DCO, the descriptive analyses comprised 4078 patients with HCC. The cohort for survival analyses comprised 3278 patients with HCC without other past or synchronous malignant diseases. ${ }^{16}$

\section{Statistics}

Data were retrieved from the Oracle database of the MCR; statistical analyses were performed by using SAS V.9.4. One-way analysis of variance and the chi-square test were used to examine

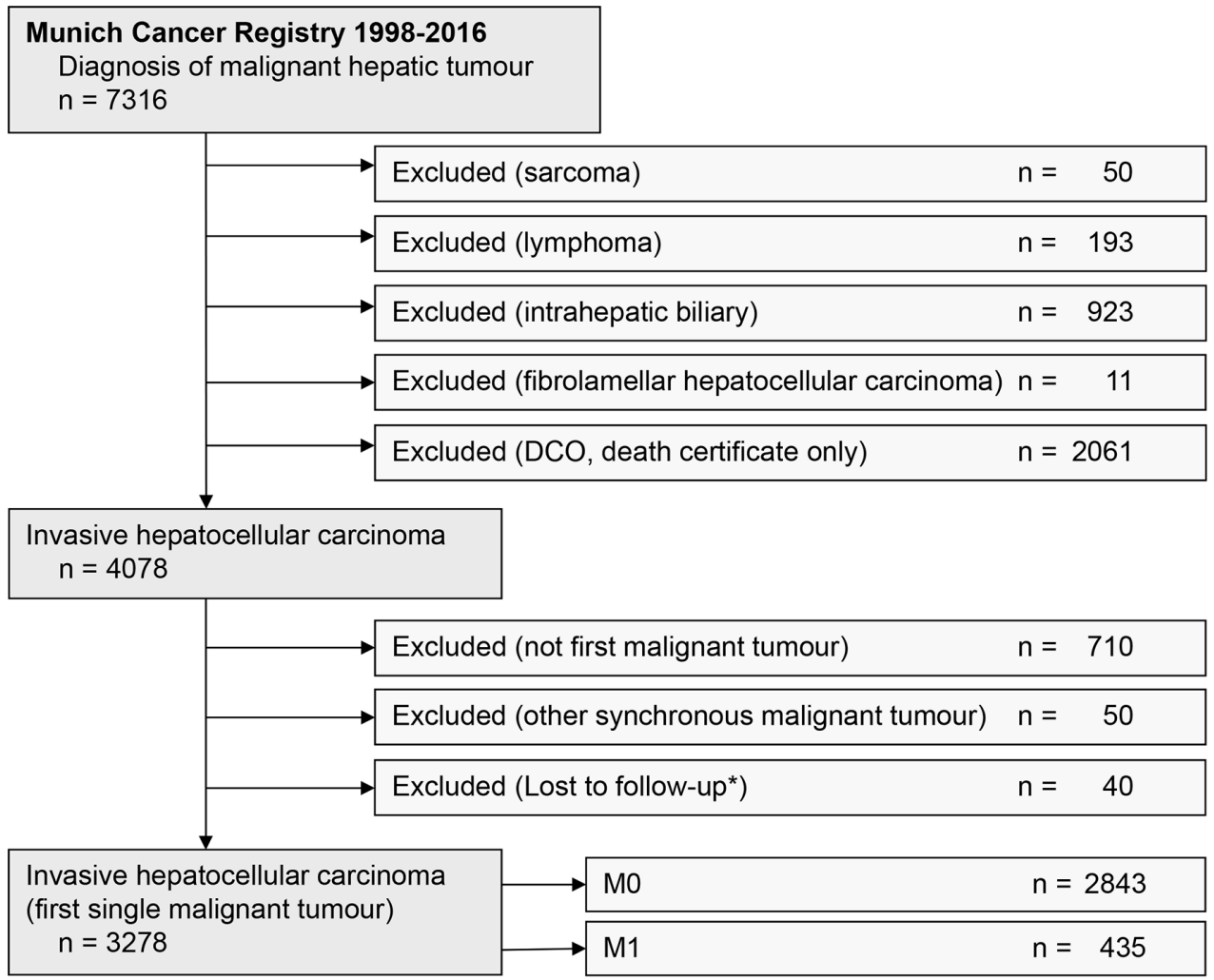

Descriptive analyses of prognostic factors

Survival analyses

* No further information after first note in in MCR

Figure 1 Study flowchart. 
continuous variables and the frequency data, respectively. The percentages of the presented subcategories were related to the sum of each item with available data; missing values were not considered. To account for competing risks, a cumulative incidence analysis was used to calculate the time to progression and differences among the subgroups were assessed by Gray's test for equality of cumulative incidence functions. ${ }^{19}$ Overall survival (OS) was estimated by the Kaplan-Meier method and was tested using the log-rank test. Relative survival (RS) was calculated by the ratio of the observed survival rate to the expected survival rate. The expected survival time of age-matched and sex-matched individuals was calculated using life tables of the general German population using the Ederer II method. ${ }^{20} \mathrm{RS}$ is regarded as estimate for cancer-specific survival in cancer registry data. The 95\% CIs were used to assess significance. Independent prognostic factors influencing survival were investigated by Cox proportional hazards models. HR and 95\% CIs are presented. Age, TNM categories and tumour grade were entered simultaneously as independent prognostic variables for multivariate analyses of survival. Time periods were included as especially interesting, independent variables. Groups of missing values of each characteristic were included in the Cox models as distinct specifications. In order to examine the effects of the time of initial diagnosis, four time periods (1998-2002, 2003-2007, 2008-2012 and 2013-2016) were defined. In parallel to the high DCO rate in HCC, there are disproportionately high quotas of missing information on tumour and treatment characteristics. For that reason, the time period can represent a surrogate parameter for the sum of unknown factors as well as for therapeutically progress with potential influence on the outcome of HCC patients. To assess the effect of age, the cut-off of 70 years was used, because the mean and median age of the analysed

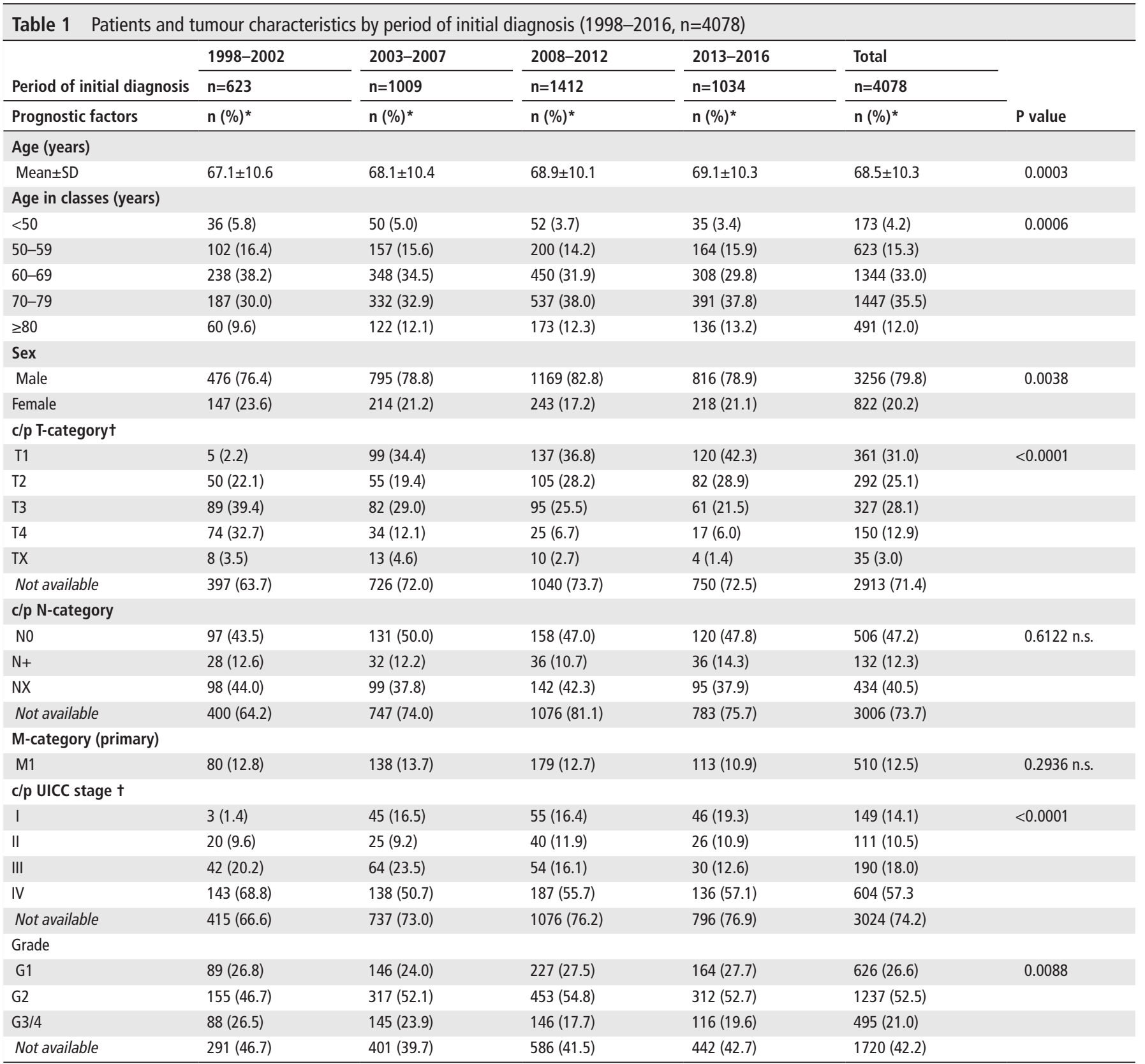

* Missing values were excluded from calculations of frequency distribution, column percentage can differ slightly from $100 \%$ due to rounding. tIn consequence of a modification of T-category in 2002 many tumours were downstaged (eg, solitary tumours without vascular invasion are classified as T1 irrespective of size). n.s., Not significant as defined by a level $\alpha$ set at 0.05 ; UICC, Union for International Cancer Control. 
A: OS (all patients, $n=3278$ )

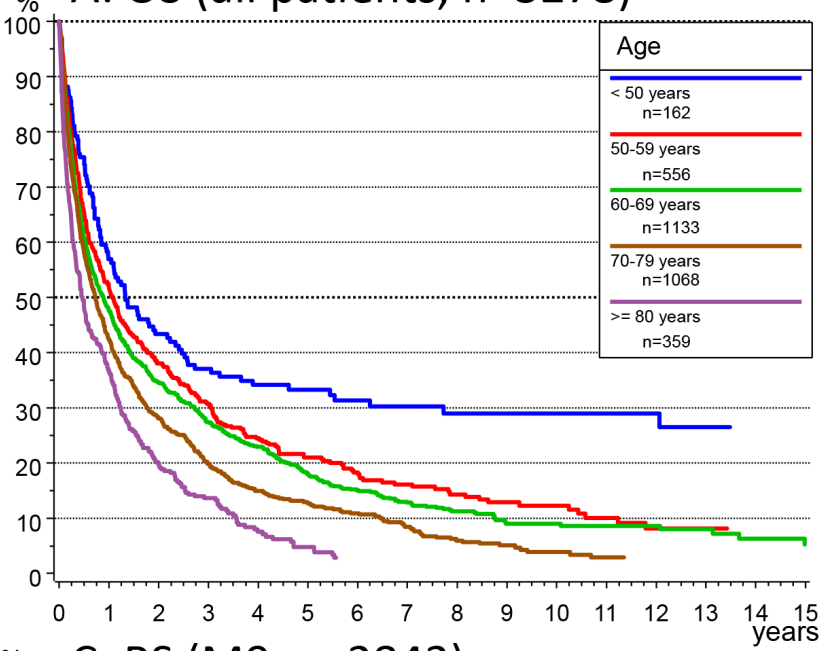

$\% \quad C: R S(M 0, n=2843)$

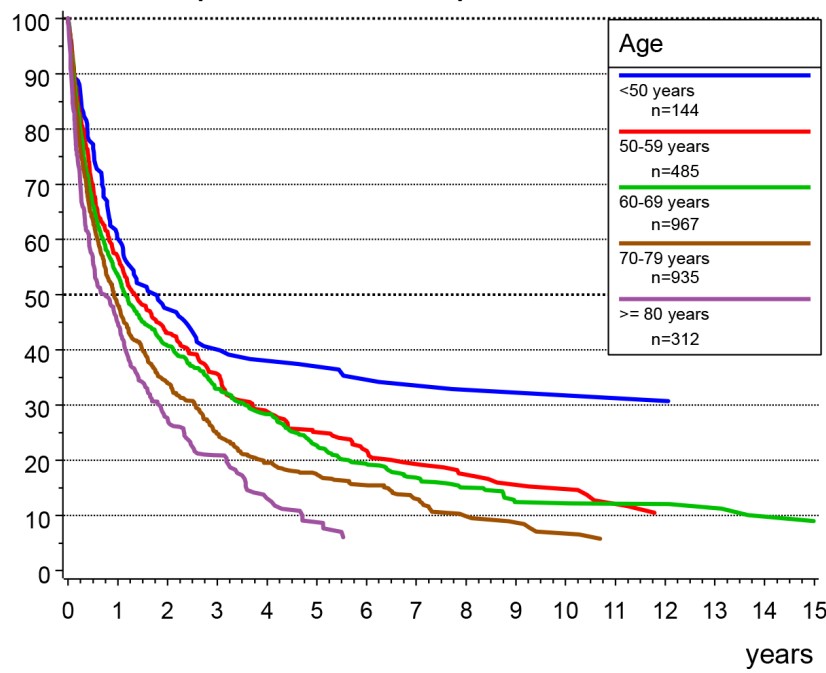

B: RS (all patients, $n=3278$ )

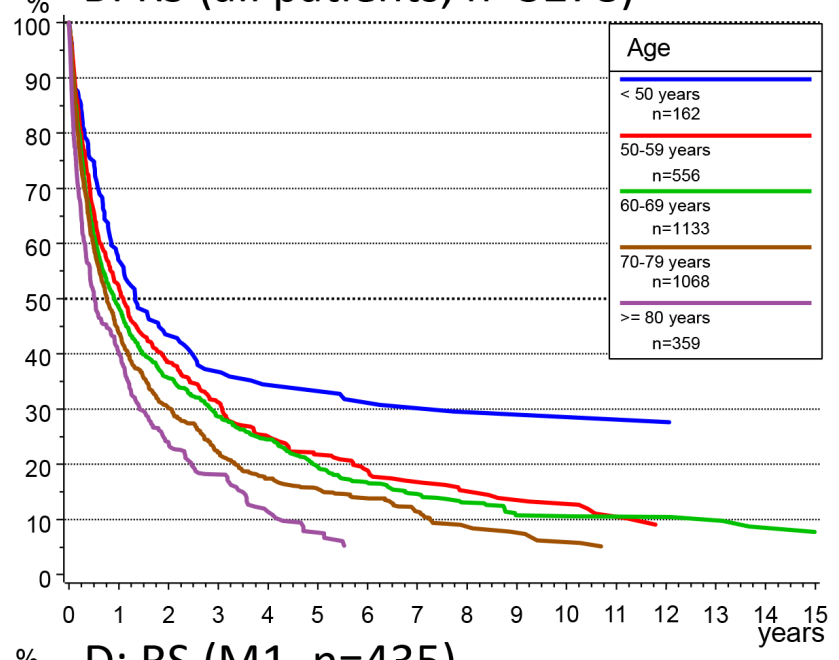

\% D: RS (M1, n=435)

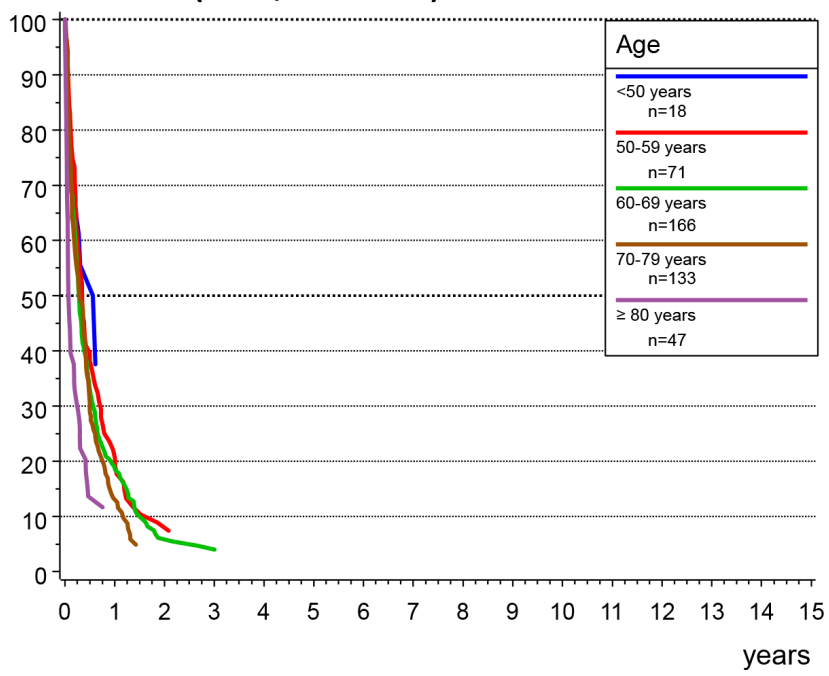

Figure 2 (A, B) Analysis of overall survival (OS) according to the Kaplan-Meier method (A) and relative survival (RS) (B) in the whole patients' collective stratified by age group. (C, D) RS by age group in patients without metastasis (M0, C) or with evidence of metastatic disease at initial diagnosis (M1, D).

cohort was 68.5 and 69.4 years, respectively. The significance level $\alpha$ was set at 0.05 in all statistical tests.

\section{RESULTS}

\section{Patient and tumour characteristics}

The age-standardised incidences of HCC per year, calculated including DCO cases and adjusted for the world standard, were 6.4 and 1.5/100 000 in men and women, respectively, and remained largely stable throughout the considered period. The examined cohort comprises 4078 cases of patients diagnosed with HCC between 1998 and 2016 (figure 1) stratified by four time periods according to the date of initial diagnosis (1998-2002, 2003-2007, 2008-2012, 2013-2016-table 1). The increase of absolute numbers of diagnoses in the time period 2003-2007 and 2008-2012 reflects the enlargement of the catchment area in year 2002 and 2007 described in the Method section. The average patients' age increased from 67.1 years in the first period to 69.1 years in the last (table 1). As expected, the vast majority of HCC patients were male $(79.8 \%)$ with the ratio of male versus female patients further increasing during the considered period ( $p=0.038$, table 1$)$. The overall percentage of available entries for tumour stage was $28.6 \%$ for the primary site of tumour (T) and $26.3 \%$ for nodal status (N), a proportion that did not change during the observation period ( $p>0.4$, data not shown). Tumour stage gradually improved over time (T1: from $34.4 \%$ to $42 \%$; T2: from $19.4 \%$ to $28.9 \%$ between 2003 and 2016) with a correlative decrease of T3 and T4 stages and a stageshift towards Union for International Cancer Control tumour stages I and II. Likewise, the percentage of grade 3-4 tumours decreased from $26.5 \%$ to $19.6 \%$ (table 1 ).

\section{Diagnostic and treatment modality}

Diagnostic liver biopsies decreased steadily from 56\% in 2004 to $30.4 \%$ in 2016 (online supplementary table 1 ). The proportion of surgical or local-ablative treatment conducted in curative intention (partial liver resection, liver transplantation and radiofrequency ablation [RFA]) increased from 33.4\% in 1998-2002 to $47.2 \%$ in $2013-2016$. However, also palliative locoregional treatment options like transarterial chemoembolisation (TACE) and selective internal radiation therapy (SIRT) were performed in an increasing fraction of patients (from $17 \%$ to $34.5 \%$ in the same time period). The frequency of systemic chemotherapeutic treatment progressively decreased until sorafenib was approved in 2008 . 

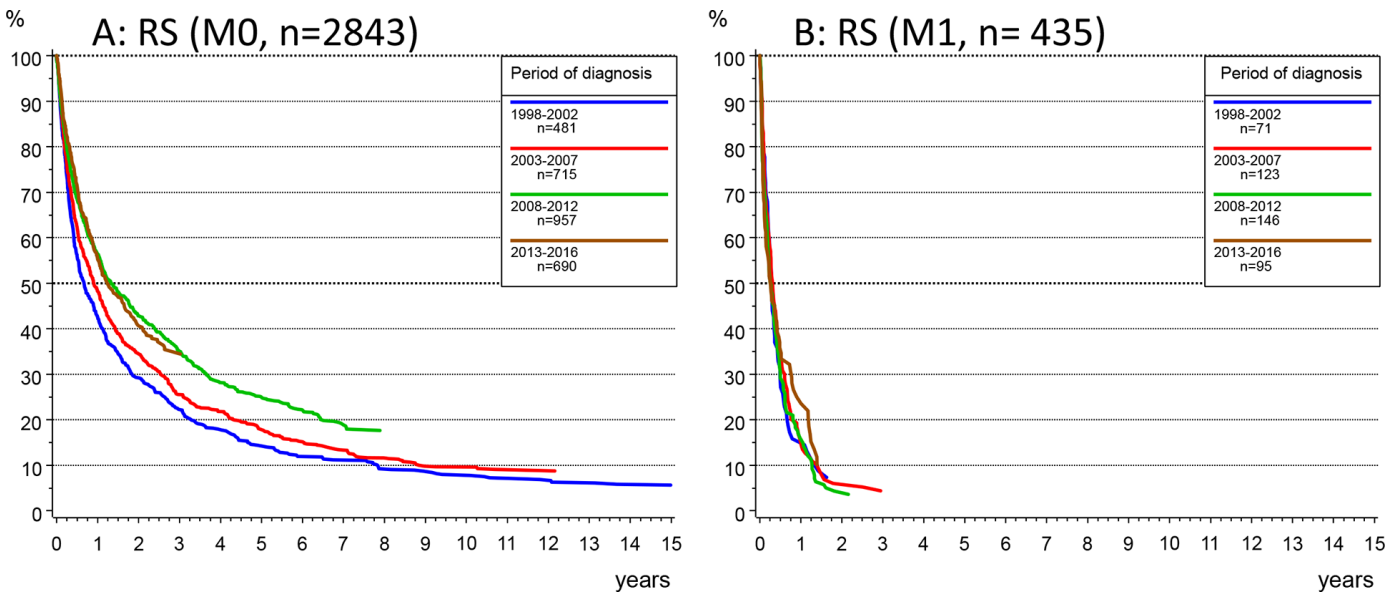

Figure 3 (A, B) Relative survival (RS) according to the Kaplan-Meier method by time period of diagnosis in patients with no metastasis (M0, A) or with metastatic disease at diagnosis (M1, B).

\section{Survival}

OS and RS rates of the total cohort were $45.5 \%$ and $47.2 \%$ at 1 year, $24.5 \%$ and $26.5 \%$ at 3 years, 16.2 and 18.4 at 5 years, and 8.2 and 10.4 at 10 years, respectively.

OS and RS stratified by age groups showed only marginal differences (figure $2 \mathrm{~A}$ and $\mathrm{B}$ ) confirming the poor disease-related outcome of HCC and the significant dependency of survival by age $(p<0.001)$. Therefore, for all subsequent analyses, only RS was presented.

In M0 patients, median survival and 3 years RS rates were 1.8 years and $40 \%$ (95\% CI $31.6 \%$ to $48.4 \%$ ) in patients younger than 50 years and 7 months and 21\% (95\% CI 15.2\% to 26.6\%) in patients older than 80 years, respectively (figure 2C). In contrast, a median survival of $<4$ months without age-related differences was observed in M1 patients (figure 2D).

Stratification by the time period of diagnosis showed that the 3 years RS in HCC increased from $19.8 \%$ in $1998-2002,22.4 \%$ in $2003-2007,30.6 \%$ in $2008-2012$ up to $31.0 \%$ in $2013-$ 2016. The median OS increased from 6 months in 1998-2002 to 12 months in 2008-2016. An improved outcome could be observed in M0 patients (figure 3A): median survival increased from 7 months in the time period 1998-2002 to 11 months in 2003-2007 and to 14 months for patients diagnosed in the two time periods since 2008 , with 3 years RS rates for the respective time periods of $22.3 \%$ (95\% CI $18.4 \%$ to $26.2 \%$ ), $25.5 \%$ and $35 \%$ (95\% CI $29.4 \%$ to $39.6 \%$ ). In contrast, survival did not improve in M1 patients (figure 3B) (with median survival $<4$ months) regardless of age at diagnosis (figure 4B).

The time period-dependent improvement of survival in M0 patients (figures $3 \mathrm{~A}$ and $4 \mathrm{~A}$ ) was not age dependent and was confirmed in the Cox regression analyses which controlled for the tumour stage at diagnosis as independent predictor of survival (table 2).

\section{DISCUSSION}

\section{Incidence and age at diagnosis}

By assessing the age-adjusted incidence of HCC in our collective, we observed a stable incidence of HCC in the last two decades. Although our analysis is based on the assessment of data from the MCR, we assume that our results are representative of the epidemiology of HCC in Germany because the age structure in Bavaria is similar to that of the German population. ${ }^{17}{ }^{18}$ This finding is at odd with the results of studies on the global trend of HCC, which consistently show a remarkable increase of the incidence of HCC worldwide. ${ }^{21}$ The discrepancy between the stable incidence of HCC observed by us and the increasing global trend is most likely due to the comparatively low prevalence of chronic HBV and HCV in Germany. Instead, previous reports on increasing incidence of HCC in Germany are likely attributable to the increasing referral of HCC patients to tertiary care centres rather than to variations of incidence in the general population. $^{22}$

Due to the advances in the treatment of $\mathrm{HCV}$, sustained virological responses to antiviral treatment have progressively increased in recent years and HCV infection has now become a virtually curable disease. ${ }^{23}$ Since $\mathrm{HCV}$ affects a disproportionally high amount of male patients, it is expected that the increasing effectiveness of antiviral treatment will cause a decrease of HCC incidence in male patients. ${ }^{24}$ However, such a trend could not be observed in our cohort yet. One possible explanation for this might be represented by the latency between HCV infection and onset of HCC and the fact that chronic HCV infection remains undetected, and thus untreated, in a large proportion of affected patients. However, it is also possible that a decline in $\mathrm{HCV}$ related HCC is counteracted by a correlative increase of HCC related to risk factors known to be increasing in the general population, which include non-alcoholic fatty liver disease (nonalcoholic steatohepatitis (NASH)), obesity and diabetes. ${ }^{25}$

The fact that the average age at diagnosis continuously increased over the observation period seems to contradict the prediction that the observed decrease of tumour stage would be accompanied by a decrease of average age at diagnosis. However, increasing age at diagnosis may in part reflect the ageing of the general population and the progressively improving effectiveness of treatment for chronic viral hepatitis, possibly resulting in a delayed onset of HCC as previously suggested. ${ }^{3}$

\section{Diagnosis, staging at diagnosis and treatment modality}

Since highly specific imaging-based diagnostic criteria for HCC were established in the early 2000s, diagnostic biopsy has become unnecessary for most patients. ${ }^{26}$ However, whether or not histology should be obtained to confirm the diagnosis of HCC or for biomarker-based patients' stratification, is still object of debate. ${ }^{27}$ In our cohort (online supplementary table 1 ), we expectedly observed a clear decline of the frequency of biopsies. However, the overall percentage of diagnostic biopsies $(45 \%)$ shows that many physicians still rely on histology for diagnosis of HCC. 

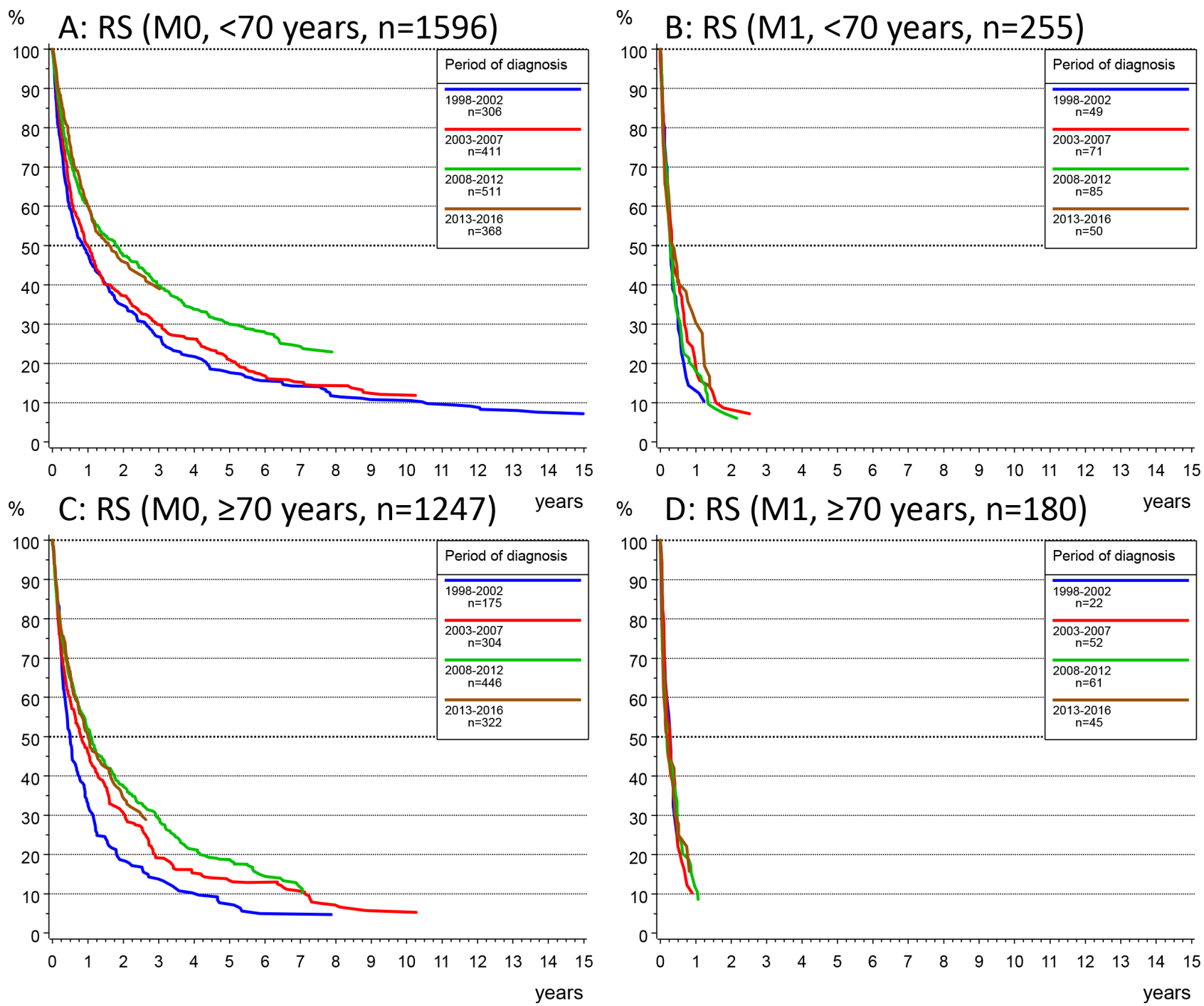

Figure 4 (A, B) Relative survival (RS) according to the Kaplan-Meier method by time period at diagnosis in patients younger than 70 years and primary non-metastatic disease $(\mathrm{M} 0, \mathrm{~A})$ or with metastasis at diagnosis $(\mathrm{M} 1, \mathrm{~B})$, and in patients aged 70 years or older with non-metastatic (M0, C) or metastatic disease (M1, D).

Surveillance by 6-monthly ultrasound examination in at-risk patients has been advised since the early 2000. Although the actual efficacy of screening programmes has been questioned, ${ }^{14} 15$ surveillance for HCC is thought to have contributed to the amelioration of the outcome of HCC patients. ${ }^{15}$ The clear shift towards lower stages and lower histological grading at diagnosis observed by us supports this notion. In fact, the amelioration of tumour stage at diagnosis could even be underrated due to the well-known stage-migration bias due to improving staging procedures. $^{28}$

Established therapeutic options with curative potential comprise ablation techniques like RFA, surgical resection and liver transplantation. TACE is used in the palliative setting for patients with HCCs confined to the liver. More recently, SIRT was employed as alternative to TACE, and since 2008 sorafenib has become an effective option for systemic treatment. ${ }^{11}$ Interestingly, the fraction of liver transplant patients in this cohort has not significantly increased compared with resection rates over the years. This is specifically interesting in the light of the fact that the allocation system has changed during this period of time and early HCC has become a privileged indication in the Model for End Stage Liver Disease (MELD) system. This may be the consequence of lower tumour stage and possibly of better liver function at diagnosis allowing for liver resection in an increasing fraction of patients. On the other hand, upfront partial hepatectomy might have been increasingly attempted due to the increasing organ shortage which represents a limiting factor for transplantation. Altogether, the percentage of patients receiving a potentially curative treatment or palliative locoregional treatment with established therapeutic relevance increased from $33.4 \%$ to $47.2 \%$ and from $17 \%$ to $34.5 \%$, respectively, showing that lower average tumour stages were associated with a growing proportion of patients benefitting from effective treatment options.

\section{Survival}

Median survival improved considerably, and doubled from 6 months in 1998-2002 to 12 months in 2008-2012 and 2013-2016. Cox regression analysis showed that tumour stage at diagnosis, tumour grading and the time period of diagnosis are independent predictors of survival whereupon time period represents a surrogate parameter for therapeutic progress with potential influence on the outcome of HCC patients (table 2). 
Table 2 Cox proportional hazard regression analyses of the association between time period of initial diagnosis and survival according to age and the presence (M1) or absence (M0) of metastasis at diagnosis (total cohort: $n=3278$, primary M0 cases: $n=2843$, primary $M 1$ cases: $n=435$ )

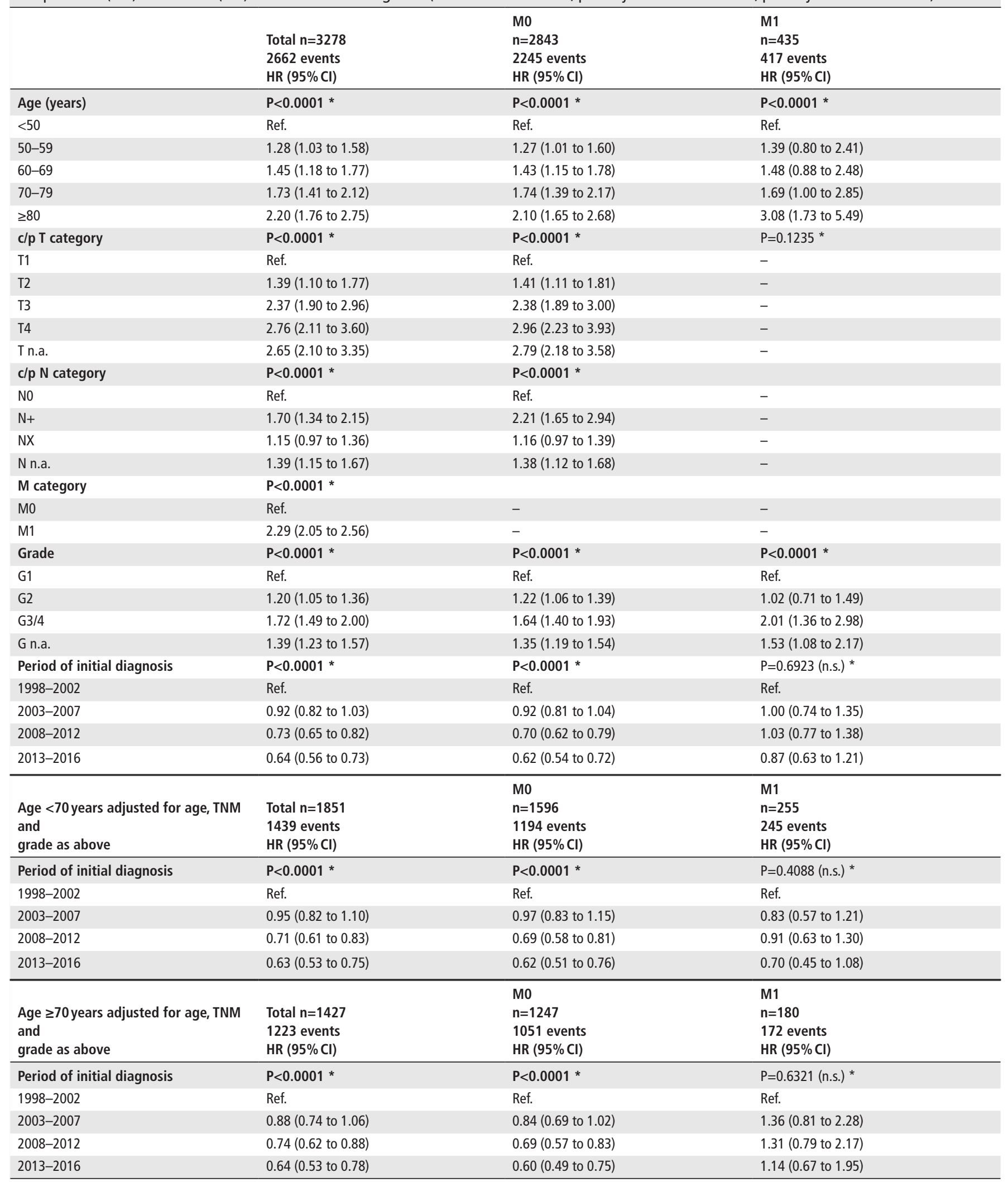

*Wald test.

n.a., Not available; n.s.: not significant as defined by a level $\alpha$ set at 0.05 ; Ref., reference; TNM, tumour, node, metastases.

The independent significance of tumour staging at diagnosis strongly highlights the importance of early diagnosis as determinant of prognosis. ${ }^{29}$ Importantly, however, the independent prognostic significance of the time period of diagnosis suggests that availability or improvement of therapeutic strategies contributed to the amelioration of survival, as well. This is likely 
to reflect the enactment of rational treatment algorithms (such as the Barcelona Clinic Liver Cancer (BCLC) algorithm, proposed in 1999), ${ }^{30}$ allowing a progressive refinement of patients' selection for different therapeutic options, including the introduction of the Milan criteria for liver transplantation, ${ }^{10}$ and restrictions to the employment of locoregional strategies. ${ }^{31}$ Therefore, patients not only benefitted from earlier tumour stage at diagnosis but also from advances in treatment modalities. ${ }^{32}$

One interesting finding of our analyses is represented by the lack of improvement in survival of patients with primary metastatic disease (figure $3 \mathrm{~B}$ ). This finding is not entirely unexpected, because therapeutic options for patients with advanced disease are still very limited. ${ }^{12}$ Sorafenib, the only evidence-based effective systemic treatment for HCC available until recently, was approved only in 2008; moreover, its employment is limited only to a small population of patients with preserved liver function despite advanced-stage HCC. Furthermore, its clinical benefit, which amounts to the modest survival advantage of about 3 months, is clearly greater in patients without extrahepatic disease as shown by subgroup analysis from the Sorafenib Hepatocellular Carcinoma Assessment Randomized Protocol (SHARP) trial. ${ }^{11} 33$ These factors explain the lack of improvement in survival of patients with metastatic disease at diagnosis and highlight the urgent need for new therapeutic options in this patients' subpopulation.

The increase of survival observed by us is not likely to have been the consequence of a lead-time bias, because earlier diagnosis was associated with an increasing percentage of patients undergoing treatment with well established, potentially creative treatment options or with treatment options which are known to be associated with a clear benefit in terms of survival and, correspondingly, with an improvement of TTP (online supplementary figure 1). ${ }^{15}$ Correspondingly, no change in the outcome of patients with metastatic disease was observed.

\section{Limitations}

A first limitation of this study is represented by the fact that this, being a longitudinal study, might be affected by several biases.

In particular, variables describing liver function are not included in our analysis. Such variables are generally not captured by cancer registry databases but are important to determine patients' stratification within the BCLC therapeutic algorithm. Therefore, we cannot estimate to which extent a possible improvement of average liver function in the patients' population (eg, owing to the improvement of antiviral treatments) might have contributed to improve the prognosis of HCC in recent years. Another limitation is given by the high proportion of patients for whom only death certificates were available (DCO cases). DCO rates for HCC in German cancer registries range between $16 \%$ and $35 \% .{ }^{345}$ DCO-related diagnoses are likely to be more frequent in patients affected by tumours with more advanced stages at diagnosis and with lower life expectancy, and who are thus less likely to have received any cancer-specific treatment. Therefore, exclusion of DCO patients from analysis of survival might cause an overestimation of survival in our collective. Nevertheless, the proportion of DCO cases in our study has decreased from 36.5\% in 1998 to 23.5\% in 2015; therefore, the observed improvement of survival estimated by time period is more likely to be underestimated than overestimated.

\section{Summary}

In summary, our results from a multicenter study in Southern Germany shows that the incidence of HCC has been stable in the last 18 years. Both implementation of screening programmes and the advances in the therapeutic stratification are likely to have contributed to improve survival of HCC patients in the general population. The fact that the prognosis of patients with metastatic disease remained unchanged highlights the unmet need for effective systemic treatment in patients with advanced HCC.

\section{Author affiliations}

${ }^{1}$ Department of Medicine II, University Hospital, Ludwig-Maximilians-University (LMU), Munich, Germany

${ }^{2}$ Munich Cancer Registry, Institute for Medical Information Processing, Biometry and Epidemiology, Ludwig-Maximilians-University (LMU), Munich, Germany

${ }^{3}$ Department of Gastroenterology, Hepatology and GI-Oncology, Hospital Bogenhausen, Munich, Germany

${ }^{4}$ Department of Internal Medicine II, Klinikum rechts der Isar, Technical University of Munich (TUM), Munich, Germany

${ }^{5}$ Department of Clinical Radiology, University Hospital, Ludwig-MaximiliansUniversity (LMU), Munich, Germany

${ }^{6}$ Department of Interventional Radiology, Klinikum Rechts der Isar, Technical University of Munich (TUM), Munich, Germany

${ }^{7}$ Department of Surgery, Klinikum Rechts der Isar, School of Medicine, Technical University of Munich (TUM), Munich, Germany

${ }^{8}$ Department of General, Visceral, Vascular and Transplant Surgery, University Hospital, Ludwig-Maximilians-University (LMU), Munich, Germany

Acknowledgements We thank all the hospitals, departments and practitioners that participated in the documentation of the data.

Contributors Study concept and design: ENDT, AS-R and JE; acquisition of data: all authors; analysis and interpretation of data: ENDT, AS-R and JE; drafting of the manuscript: ENDT and AS-R; critical revision of the manuscript for important intellectual content: all authors; statistical analysis: ENDT, AS-R and JE.

Funding The authors have not declared a specific grant for this research from any funding agency in the public, commercial or not-for-profit sectors.

Competing interests None declared.

Patient consent for publication Not required.

Provenance and peer review Not commissioned; externally peer reviewed.

Open access This is an open access article distributed in accordance with the Creative Commons Attribution Non Commercial (CC BY-NC 4.0) license, which permits others to distribute, remix, adapt, build upon this work non-commercially, and license their derivative works on different terms, provided the original work is properly cited, appropriate credit is given, any changes made indicated, and the use is non-commercial. See: http://creativecommons.org/licenses/by-nc/4.0/.

\section{ORCID iD}

Enrico N De Toni http://orcid.org/0000-0002-8101-8207

\section{REFERENCES}

1 Singal AG, El-Serag HB. Hepatocellular carcinoma from epidemiology to prevention: translating knowledge into practice. Clin Gastroenterol Hepatol 2015;13:2140-51.

2 Rich NE, Hester C, Odewole M, et al. Racial and ethnic differences in presentation and outcomes of hepatocellular carcinoma. Clin Gastroenterol Hepatol 2019;17:551-9.

3 Weinmann A, Koch S, Niederle IM, et al. Trends in epidemiology, treatment, and survival of hepatocellular carcinoma patients between 1998 and 2009: an analysis of 1066 cases of a German HCC Registry. J Clin Gastroenterol 2014;48:279-89.

4 Erhardt A, Zhu E, Blondin D, et al. [Increasing number and improved survival of patients with hepatocellular carcinoma from 1988 to 2007: data of a German university clinic]. Z Gastroenterol 2011;49:720-7.

5 Schütte K, Kipper M, Kahl S, et al. Clinical characteristics and time trends in etiology of hepatocellular cancer in Germany. Digestion 2013;87:147-59.

6 Liang TJ, Block TM, McMahon BJ, et al. Present and future therapies of hepatitis B: From discovery to cure. Hepatology 2015;62:1893-908.

7 Vermehren J, Park JS, Jacobson IM, et al. Challenges and perspectives of direct antivirals for the treatment of hepatitis C virus infection. J Hepatol 2018;69:1178-87.

8 Gerbes A, Zoulim F, Tilg H, et al. Gut roundtable meeting paper: selected recent advances in hepatocellular carcinoma. Gut 2018;67:380-8.

9 Berhane S, Toyoda H, Tada T, et al. Role of the GALAD and BALAD-2 Serologic Models in Diagnosis of Hepatocellular Carcinoma and Prediction of Survival in Patients. Clin Gastroenterol Hepatol 2016;14:875-86.

10 Mazzaferro V, Regalia E, Doci R, et al. Liver transplantation for the treatment of small hepatocellular carcinomas in patients with cirrhosis. N Engl J Med 1996;334:693-700. 
11 Llovet JM, Ricci S, Mazzaferro V, et al. Sorafenib in advanced hepatocellular carcinoma. N Engl J Med 2008;359:378-90.

12 European Association for the Study of the Liver. Electronic address: easloffice@ easloffice.euEuropean Association for the Study of the Liver. EASL Clinical Practice Guidelines: Management of hepatocellular carcinoma. J Hepatol 2018;69:182-236.

13 Sato $M$, Tateishi $R$, Yasunaga $H$, et al. Association between hospital volume and inhospital mortality following radiofrequency ablation for hepatocellular carcinoma. BJS Open 2017;1:50-4.

14 Moon AM, Weiss NS, Beste LA, et al. No association between screening for hepatocellular carcinoma and reduced cancer-related mortality in patients with cirrhosis. Gastroenterology 2018;155:1128-39.

15 Cucchetti A, Trevisani F, Pecorelli A, et al. Estimation of lead-time bias and its impact on the outcome of surveillance for the early diagnosis of hepatocellular carcinoma. J Hepatol 2014;61:333-41.

16 Registry MC. Functions and structure, catchment area, cancer statistics, ICD-10 C22 Liver cancer, Incidence and mortality. Munich, Bavaria, Germany. 2018 https://www. tumorregister-muenchen.de/en/index.php

17 Destatis Statistisches Bundesamt. German Population by Age-Groups. 2019 https:// www.destatis.de/EN/FactsFigures/SocietyState/Population/CurrentPopulation/Tables_/ Irbev01.html

18 Bayerisches Landesamt für Statistik. Demographisches Profil für den Regierungsbezirk Oberbayern. 2018 https://www.statistik.bayern.de/statistik/byrbz/

19 Kalbfleisch JD, Prentice RL. The statistical analysis of failure time data. New York: John Wiley and Sons, Inc, 1980.

20 Ederer $\mathrm{F}$, Heise $\mathrm{H}$. Instructions to IBM 650 programmers in processing survival computations. Technical, end results evaluation section. Bethesda, MD, USA: National Cancer Institute, 1959.

21 Bertuccio P, Turati F, Carioli G, et al. Global trends and predictions in hepatocellular carcinoma mortality. J Hepato/ 2017;67:302-9.

22 Schurr R, Stölzel U, Schuppan D, et al. [Increased incidence of hepatocellular and intrahepatic cholangiocellular carcinoma in northeast Germany]. Dtsch Med Wochenschr 2006;131:1649-55.

23 Kanwal F, Kramer J, Asch SM, et al. Risk of Hepatocellular Cancer in HCV Patients Treated With Direct-Acting Antiviral Agents. Gastroenterology 2017;153:996-1005.
24 Baden R, Rockstroh JK, Buti M. Natural history and management of hepatitis C: does sex play a role? J Infect Dis 2014;209(Suppl 3):S81-5.

25 Estes C, Anstee QM, Arias-Loste MT, et al. Modeling NAFLD disease burden in China, France, Germany, Italy, Japan, Spain, United Kingdom, and United States for the period 2016-2030. J Hepatol 2018;69:896-904.

26 Bruix J, Sherman M, Llovet JM, et al. Clinical management of hepatocellular carcinoma. Conclusions of the Barcelona-2000 EASL conference. European Association for the Study of the Liver. Journal of hepatology 2001;35:421-30.

27 Mueller C, Waldburger N, Stampfl U, et al. Non-invasive diagnosis of hepatocellular carcinoma revisited. Gut 2018;67:991-3.

28 Dinan MA, Curtis LH, Carpenter WR, et al. Stage migration, selection bias, and survival associated with the adoption of positron emission tomography among medicare beneficiaries with non-small-cell lung cancer, 1998-2003. J Clin Oncol 2012;30:2725-30.

29 Simmons OL, Feng Y, Parikh ND, et al. Primary Care Provider Practice Patterns and Barriers to Hepatocellular Carcinoma Surveillance. Clin Gastroenterol Hepatol 2019;17.

30 Llovet JM, Brú C, Bruix J. Prognosis of hepatocellular carcinoma: the BCLC staging classification. Semin Liver Dis 1999;19:329-38.

31 White JA, Gray SH, Li P, et al. Current guidelines for chemoembolization for hepatocellular carcinoma: room for improvement? Hepatol Commun 2017; 1:338-46.

32 Khalaf N, Ying J, Mittal S, et al. Natural History of Untreated Hepatocellular Carcinoma in a US Cohort and the Role of Cancer Surveillance. Clin Gastroenterol Hepatol 2017;15:273-81

33 Bruix J, Cheng AL, Meinhardt G, et al. Prognostic factors and predictors of sorafenib benefit in patients with hepatocellular carcinoma: Analysis of two phase III studies. J Hepatol 2017;67:999-1008.

34 Vohmann C, Urbschat I, Sirri E, et al. Krebs in Niedersachsen 2015 [online book (pdf)]. Hannover, Niedersachen, Germany. 2018 www.krebsregister-niedersachsen.de/ dateien/jahresberichte/jb2015/EKN\%20Jahresbericht\%202015_Gesamt.pdf.

35 Krebsregister Rheinland-Pfalz. Bericht 2017. Leber (C22) Mainz, Rheinland-Pfalz, Germany. 2017 https://www.krebsregister-rlp.de/veroeffentlichungen/auswertungeneinzelner-krebsarten/jahr-2017/2017. 